Arch. Tierz., Dummerstorf 46 (2003) 1, 77-84

Aus dem Institut für Tierzucht und Tierhaltung mit Tierklinik der Martin-Luther-Universität Halle-Wittenberg ${ }^{1}$, der Universität Lubljana ${ }^{2}$, Slowenien, der FAL Mariensee ${ }^{3}$ und der Lohmann Tierzucht GmbH, Cuxhaven ${ }^{4}$

NORBERT MIELENZ ${ }^{1}$, MILENA KOVAC ${ }^{2}$, EILDERT GROENEVELD ${ }^{3}$, RUDOLF PREISINGER ${ }^{4}$, MATHIAS SCHMUTZ ${ }^{4}$ und LUTZ SCHÜLER ${ }^{1}$

\title{
Genetische Parameter für Merkmale der Eiproduktion geschätzt mit additiven und Dominanzmodellen bei Legehennen
}

\begin{abstract}
Summary
Title of the paper: Genetic evaluation of egg production traits based on additive and dominance models in laying hens

The study was conducted to estimate additive and dominance variances for egg production traits of two commercial purebred lines (A \& D) of laying hens using an animal model analysis. Breeding values estimated from a dominance model were compared to those from an additive model.

In total, 8625 records of line A and 8968 records of line D from three generations were used in the analysis. Every record contained information on the number of eggs laid between 20 and 28 (EN1-2), 28 and 48 (EN3-7) and between 20 and 48 (EN1-7) weeks of age. Further average egg weight (EW), egg weight at 28 (EW1), 33 (EW2) and 40 (EW3) weeks of age were available. An additive animal model and one- and three-trait dominance models including the inbreeding coefficients as covariates were fitted to the data. Simultaneous estimates of the additive and dominance variance components were obtained using the REML method. The estimates of $h^{2}$ from the dominance models for EN1-2, EN3-7, EN1-7 and EW were 0.40, 0.15, 0.28 and 0.62 for line A and 0.38, $0.16,0.26$ and 0.53 for line $\mathrm{D}$. The ratios $\mathrm{d}^{2}$ of the dominance variance to total variance were moderate for EN (0.12-0.13) and low for EW (0.04-0.07) within line A but relatively low for EN (0.03-0.12) and high for EW $(0.12-0.21)$ within line D. Between the breeding values of the best 100 hens of each generation estimated with the additive and the dominance models for EN1-2, EN3-7, EN1-7 and EW high rank correlations (0.946, 0.896, 0.945 and 0.991 in line A and 0.996, 0.899, 0.971 and 0.951 in line D were found.
\end{abstract}

Key Words: hens, egg production, dominance variation, breeding value estimation

\section{Zusammenfassung}

Das Ziel der Untersuchungen bestand in der Schätzung von additiver und Dominanzvarianz für Merkmale der Eiproduktion von zwei Reinzuchtlinien (A \& D) eines kommerziellen Zuchtprogrammes für weißschalige Legehennen und im Vergleich der Zuchtwerte geschätzt mit rein-additiven und um Dominanz erweiterte Tiermodelle. Insgesamt 8625 Datensätze von Linie A und 8968 Datensätze von Linie D aus drei Generationen wurden analysiert. Jeder Datensatz enthielt die Anzahl der gelegten Eier zwischen 20 und 28 (EN1-2), 28 und 48 (EN3-7) und zwischen 20 und 48 (EN1-7) Lebenswochen. Weiterhin standen die Eigewichte in Lebenswoche 28 (EW1), 33 (EW2), 40 (EW3) und das mittlere Eigewicht (EW) aus allen drei Messzeitpunkten zur Verfügung. Zur Schätzung der genetischen Parameter wurden Ein- und Dreimerkmalsmodelle mit und ohne Dominanz unter Einbeziehung der Inzuchtkoeffizienten als Kovariable bei Verwendung der REML-Methode ausgewertet. Die Schätzungen von $\mathrm{h}^{2}$ resultierend aus dem Dominanzmodell für die Merkmale EN1-2, EN3-7, EN1-7 und EW waren $0,40,0,15,0,28$ und 0,62 in Linie A und 0,38, 0,16, 0,26 und 0,53 in Linie D. Die Anteile $\mathrm{d}^{2}$ der Dominanzvarianz an der phänotypischen Gesamtvarianz waren in Linie A moderat für die Legeleistung $(0,12-$ $0,13)$ und gering für das Eigewicht $(0,04-0,07)$. Dagegen zeigte Linie D hohe $\mathrm{d}^{2}$-Werte für das Eigewicht $(0,12-$ $0,21)$ und relativ geringe $d^{2}$-Werte für die Eizahl $(0,03-0,12)$. Zwischen den Zuchtwerten der besten 100 Tiere aus jeder Generation geschätzt mit und ohne Berücksichtigung von Dominanz ergaben sich für EN1-2, EN3-7, EN1-7 und EW hohe Rangkorrelationen von 0,946, 0,896, 0,945 und 0,991 in Linie A und von 0,996, 0,899, 0,971 und 0,951 in Linie D.

Schlüsselwörter: Hennen, Eiproduktion, Dominanzvarianz, Zuchtwertschätzung 
1.

Einleitung

Die Schätzung der Dominanzvariation in einer Population ist aus folgenden Gründen wünschenswert: Bereitstellung einer unverzerrten Schätzung für die Heritabilität im engeren Sinn, Ableitung genauerer Vorhersagen für die additiven Effekte und Verwendung der Dominanzeffekte bei der Optimierung von Kreuzungsprogrammen oder von speziellen Verpaarungsstrategien. Erste Untersuchungen zur Schätzung der Dominanzvarianz mit der REML-Methode bei Unterstellung eines Tiermodells gehen auf WEI und VAN DER WERF (1993) zurück. In den letzten Jahren wurden zunehmend additive Tiermodelle, erweitert um Dominanzeffekte, auch für praxisrelevante Tierumfänge bei den Tierarten Schwein und Rind (MISZTAL, 1997, MISZTAL u.a., 1998) angewendet. Die direkte Aufstellung der Inversen der Verwandtschaftsmatrix $\mathrm{D}^{-1}$ erfolgt mit den von HOESCHELE und VAN RADEN (1991) abgeleiteten Algorithmen. Zur effektiven Berechnung von $\mathrm{D}^{-1}$ muss eine nicht ingezüchtete Population vorausgesetzt werden. Inzucht verkompliziert die genetische Kovarianzstruktur in einer Population bei Anwesenheit von Dominanz erheblich. So erfordert die Berechnung der genetischen Kovarianz zwischen verwandten Tieren unter anderem die Schätzung der Kovarianz zwischen additiven und Dominanzeffekten sowie die Kenntnis der Inzuchtdepression (UIMARI und MÄKI-TANILA, 1992; LANGE, 1997). Durch Einbeziehung der Inzuchtkoeffizienten aller Tiere über eine Regression versucht man die Approximation mit Dominanzmodellen weiter zu verbessern (vgl. DE BOER, 1994). Neben der Schätzung des Anteiles der Dominanzvarianz an der Gesamtvarianz erfolgten parallel Untersuchungen zum Einfluss der Dominanz auf die geschätzten Zuchtwerte beim Übergang von additiven zu Dominanzmodellen (VARONA u.a., 1998). Neuere Arbeiten zur Schätzung der Dominanzvariation beim Geflügel unter Einbeziehung von Kreuzungsleistungen sind bei BESBES und GIBSON (1999) zu finden. Ergebnisse für Dominanzmodelle mit simultaner Einbeziehung mehrerer Merkmale liegen bisher nicht vor.

Für die Auswertung standen Daten von zwei Linien der Rasse Weißes Leghorn, welche Hahnen- und Hennenlinie in einem Kreuzungsprogramm repräsentieren, zur Verfügung. Leistungsdaten der überlebenden Hennen aus 3 Generationen wurden für die Analysen aufbereitet. Die untersuchten Merkmale waren:

(a) Anzahl der Eier gelegt zwischen 20 bis 28 (EN1-2), zwischen 28 bis 48 (EN37) und zwischen 20 bis 48 (EN1-7) Lebenswochen.

(b) Eigewicht in Lebenswoche 28 (EW1), 33 (EW2), 40 (EW3) und mittleres Eigewicht aus den drei Messzeitpunkten (EW) .

Anhaltspunkte über die Populationsstruktur und das Leistungsniveau der Linien liefert Tabelle 1.

Tabelle 1

Mittelwerte $(\overline{\boldsymbol{x}})$ und Standardabweichungen (SD) für ausgewählte Merkmale der Eiproduktion (Mean values $(\overline{\boldsymbol{X}})$ and standard deviations (SD) of egg production traits)

\begin{tabular}{l|ccc|cc|cc|cc}
\hline & \multicolumn{3}{|c|}{ Datenstruktur } & \multicolumn{2}{c|}{ EN1-2 } & \multicolumn{2}{c}{ EN3-7 } & \multicolumn{2}{c}{ EW (g) } \\
Linie & $\mathbf{N}$ & Väter & Mütter & $\overline{\boldsymbol{x}}$ & SD & $\overline{\boldsymbol{x}}$ & $\mathbf{S D}$ & $\overline{\boldsymbol{x}}$ & SD \\
\hline A & 8625 & 236 & 1756 & 32,0 & 11,2 & 129,3 & 8,01 & 59,7 & 3,69 \\
D & 8968 & 235 & 1969 & 35,3 & 10,7 & 129,9 & 7,68 & 58,8 & 3,25 \\
\hline
\end{tabular}




\section{$2.1 \quad$ Modelle}

Die Schätzung der additiven und der Dominanzvarianz in beiden Linien basierte auf dem folgenden Modell:

(1) $\quad y=X \beta+\Delta b+Z a+Z W f+e$

Hierbei sind: $y$ - Vektor der Beobachtungen für die Eiproduktion, $\beta$ - Vektor der kombinierten fixen Effekte von Generation (bzw. Jahr), Haus, Schlupf und Etage, $\Delta$ Inzuchtdepression, $b$ - Vektor der Inzuchtkoeffizienten, $f$ - Vektor der elterlichen Dominanzeffekte (Vollgeschwister-Familieneffekte), $X, Z$ and $W$ - Versuchsplanmatrizen, zur Verknüpfung der Effekte mit den Beobachtungen. Die Schätzung der genetischen Parameter erfolgte sowohl unter Verwendung von Ein- und Dreimerkmalsmodellen mit der REML-Methode unter Nutzung von Programmpaket VCE-5 (KOVAC u.a., 2002).

\section{$2.2 \quad$ Transformation der Daten}

Die Anwendung der REML-Methode zur Varianzkomponentenschätzung(VKS) setzt unter anderem Normalverteilung für die Resteffekte in gemischten linearen Modellen der Gestalt (1) voraus. Diese Annahme lässt sich zumindest für die Legeleistung nur unzureichend erfüllen. Die Merkmale EN3-7 und EN1-7 genügen einer linksschiefen (rechtssteilen) Verteilung während die kumulative Eizahl in den ersten 2 Legemonaten (EN1-2) einer rechtsschiefen (linkssteilen) Verteilung folgt. Folgerichtig existieren Arbeiten, in denen vor der VKS für das Merkmal Legeleistung eine Transformation auf Normalverteilung durchgeführt wurde (WEI und VAN DER WERF, 1993; KOERHUIS, 1996; BESBES und GIBSON, 1999). Für die Legeleistung hat sich eine Variante der Potenztransformation (BOX und COX, 1964) als vorteilhaft erwiesen, deren Anwendung auf Geflügeldaten bei IBE und HILL (1988) ausführlich demonstriert wird. Am untersuchten Datenmaterial wurde ebenfalls eine Potenztransformation durchgeführt. Der optimale t-Wert wurde so gewählt, dass die Restvarianz in einem Modell mit fixen Vater- und Muttereffekten minimal ausfällt. Zusätzlich wurde die von JARQUE und BERA (1980) vorgeschlagene Statistik

(2) $\quad \boldsymbol{J B}=\boldsymbol{N} \cdot\left(\frac{1}{6} \cdot \gamma_{1}+\frac{1}{24} \cdot \gamma_{2}\right)$

bestimmt, wobei $N$ die Anzahl der Beobachtungen, $\gamma_{1}$ die Schiefe und $\gamma_{2}$ der Exzess der zufälligen Resteffekte sind. Die Ergebnisse der Transformation sind in Tabelle 2 aufgelistet, wobei $d^{2}$ den Anteil der Dominanzvarianz an der phänotypischen Gesamtvarianz darstellt.

Tabelle 2

Optimale t-Werte der Potenztransformation bei Verwendung von 2 empirischen Kriterien einschließlich der zugehörigen $\mathrm{h}^{2}$ - und $\mathrm{d}^{2}$-Werte (Optimal t-values of power transformation using two empirical criterions including the corresponding $\mathrm{h}^{2}$ - and $\mathrm{d}^{2}$-values)

\begin{tabular}{|c|c|c|c|c|c|c|c|c|c|}
\hline \multirow[b]{2}{*}{ Line } & \multirow[b]{2}{*}{ trait } & \multicolumn{2}{|c|}{ untransformiert } & \multicolumn{3}{|c|}{$\sigma_{e}^{2} \rightarrow$ Min! } & \multicolumn{3}{|c|}{$J B \rightarrow$ Min! } \\
\hline & & $\mathbf{h}^{2}$ & $d^{2}$ & t & $\mathbf{h}^{2}$ & $d^{2}$ & t & $\mathbf{h}^{2}$ & $d^{2}$ \\
\hline \multirow[t]{3}{*}{$\bar{A}$} & EN1-2 & 0,40 & 0,13 & 0,7 & 0,40 & $\overline{0,14}$ & 0,8 & 0,40 & 0,14 \\
\hline & EN1-7 & 0,28 & 0,12 & 2,9 & 0,30 & 0,11 & 4,1 & 0,31 & 0,11 \\
\hline & EN3-7 & 0,15 & 0,13 & 9,0 & 0,19 & 0,15 & 12,0 & 0,19 & 0,15 \\
\hline \multirow[t]{3}{*}{ D } & EN1-2 & 0,38 & 0,03 & 0,8 & 0,38 & 0,03 & 0,4 & 0,36 & 0,04 \\
\hline & EN1-7 & 0,26 & 0,09 & 2,7 & 0,27 & 0,08 & 3,2 & 0,28 & 0,08 \\
\hline & EN3-7 & 0,16 & 0,12 & 9,7 & 0,19 & 0,13 & 14,1 & 0,20 & 0,12 \\
\hline
\end{tabular}

Durch die Transformation erhöhen sich die geschätzten $\mathrm{h}^{2}$-Werte nur geringfügig während die $d^{2}$-Werte annähernd gleich bleiben. Durch Einbeziehung von fixen Haus-, 
Schlupf- und Etageneffekten konnte für das Merkmal Legeleistung bereits eine wesentliche Verbesserung der Verteilungseigenschaften erreicht werden. Aufgrund der tWerte nahe Eins ist eine Transformation für das Merkmal EN1-2 nicht notwendig. Für das Merkmal EN1-7 mit moderaten t-Werten von 2,9 in Linie A und 2,7 in Linie D scheint eine Transformation ebenfalls nicht zwingend. Einerseits aus Gründen der Interpretierbarkeit insbesondere der geschätzten Zuchtwerte und andererseits um den Vergleich der Linien zu gewährleisten wurde deshalb auf eine Transformation vollständig verzichtet.

\section{Dominanzvariation}

Die Schätzungen für die Heritabilitäten und für die Anteile der Dominanzvarianzen an den Gesamtvarianzen auf der Grundlage von Einmerkmalsmodellen gemäß (1) sind in Tabelle $3 \mathrm{zu}$ finden.

Tabelle 3

Schätzung der genetischen Parameter mit rein-additiven ( $h^{2}\left(\right.$ add.)) und Dominanzmodellen $\left(\mathrm{h}^{2}\right.$ und $\left.\mathrm{d}^{2}\right)$ bei Berücksichtigung von Inzucht (Estimation of genetic parameters with additive $h^{2}$ (add.) and dominance $\left(h^{2}\right.$ and $\left.d^{2}\right)$ models including inbreeding)

\begin{tabular}{l|c|c|c|c|c|c}
\hline & \multicolumn{3}{|c|}{ Linie A } & \multicolumn{3}{c}{ Linie D } \\
\hline Merkm. & $\mathbf{h}^{2}$ (add.) & $\mathbf{h}^{2} \pm \mathbf{s}$ & $\mathbf{d}^{2} \pm \mathbf{s}$ & $\mathbf{h}^{\mathbf{2}}$ (add.) & $\mathbf{h}^{2} \pm \mathbf{~ s}$ & $\mathbf{d}^{2} \pm \mathbf{~}$ \\
\hline EN1-2 & $0,45 \pm, 019$ & $0,40 \pm, 021$ & $0,13 \pm, 025$ & $0,39 \pm, 019$ & $0,38 \pm, 019$ & $0,03 \pm, 023$ \\
EN3-7 & $0,20 \pm, 016$ & $0,15 \pm, 017$ & $0,13 \pm, 027$ & $0,21 \pm, 019$ & $0,16 \pm, 016$ & $0,12 \pm, 025$ \\
EN1-7 & $0,33 \pm, 019$ & $0,28 \pm, 019$ & $0,12 \pm, 025$ & $0,30 \pm, 019$ & $0,26 \pm, 017$ & $0,09 \pm, 025$ \\
\hline EW & $0,63 \pm, 018$ & $0,62 \pm, 022$ & $0,06 \pm, 023$ & $0,58 \pm, 019$ & $0,53 \pm, 018$ & $0,17 \pm, 025$ \\
EW1 & $0,51 \pm, 019$ & $0,48 \pm, 021$ & $0,06 \pm, 025$ & $0,47 \pm, 019$ & $0,41 \pm, 018$ & $0,17 \pm, 027$ \\
EW2 & $0,43 \pm, 018$ & $0,41 \pm, 020$ & $0,07 \pm, 023$ & $0,45 \pm, 019$ & $0,37 \pm, 018$ & $0,21 \pm, 028$ \\
EW3 & $0,48 \pm, 019$ & $0,46 \pm, 021$ & $0,04 \pm, 022$ & $0,45 \pm, 019$ & $0,40 \pm, 019$ & $0,12 \pm, 026$ \\
\hline
\end{tabular}

Innerhalb Linie A wurden für die Legeleistungen Anteile der Dominanzvarianz an der Gesamtvarianz von 12 bis 13\% und für die Eigewichte von 4 bis 7\% gefunden. Für die Linie D ergab sich ein konträres Bild. Hier lagen die d $\mathrm{d}^{2}$-Werte zwischen 3 und 12\% für die Eizahl und zwischen 12 und 21\% für die Eigewichte. Ein Vergleich der Heritabilitäten geschätzt mit rein-additiven und mit Modellen erweitert um Dominanzeffekte lieferte eine generelle Verminderung. Die $\mathrm{h}^{2}$-Werte basierend auf dem Dominanzmodell reduzierten sich um 11, 25, 16 und 3\% für EN1-2, EN3-7, EN1-7 und EW in Linie A und um 3, 25, 12 und 9\% in Linie D. Die geschätzten Dominanzanteile für Linie A entsprechen den Untersuchungsergebnissen von WEI und VAN DER WERF (1993) und von BESBES und GIBSON (1999). Dagegen zeigt Linie D relativ hohe Dominanzanteile für das Merkmal Eigewicht. Ursachen könnten die Stellung dieser Linien im Hybridzuchtprogramm sowie unterschiedliche Selektionsintensitäten und Selektionsziele im Verlauf der züchterischen Bearbeitung beider Linien sein. Als Tendenz lässt sich festhalten: In dem Merkmal, in welchem eine Linie beim Mittelwertsvergleich Überlegenheit zeigt, tritt weniger Dominanzvariation auf.

Die neue Version 5 von VCE gestattet erstmalig die Schätzung von genetischen $\mathrm{Pa}-$ rametern mit Mehrmerkmalsmodellen bei Einbeziehung tierspezifischer Dominanzeffekte. Die genetischen Korrelationen geschätzt mit einem rein-additiven Modell $\left(\mathrm{r}_{\mathrm{g}}(\mathrm{add})\right)$ sind den Schätzungen für die additiv-genetischen $\left(\mathrm{r}_{\mathrm{a}}\right)$ und die dominanz-genetischen Korrelationen $\left(r_{d}\right)$ auf der Grundlage von 3-Merkmalsmodellen in Tabelle 4 gegenübergestellt. 
Tabelle 4

Genetische Korrelationen geschätzt bei Verwendung von 3-Merkmalsmodellen mit und ohne Dominanz (Genetic correlations estimated using three-trait models with and without dominance)

\begin{tabular}{l|c|c|c|c|c|c}
\hline & \multicolumn{3}{|c|}{ Linie A } & \multicolumn{3}{c}{ Linie D } \\
\hline Merk1:Merk2 & $\mathbf{r}_{\mathbf{g}}$ (add.) & $\mathbf{r}_{\mathbf{a}} \pm \mathbf{s}$ & $\mathbf{r}_{\mathbf{d}} \pm \mathbf{s}$ & $\mathbf{r}_{\mathbf{g}}$ (add.) & $\mathbf{r}_{\mathbf{a}} \pm \mathbf{s}$ & $\mathbf{r}_{\mathbf{d}} \pm \mathbf{s}$ \\
\hline EN1-2:EN3-7 & 0,08 & $0,08 \pm, 029$ & $0,15 \pm, 054$ & 0,01 & $-0,04 \pm, 058$ & $0,65 \pm, 382$ \\
EN1-2:EW & $-0,25$ & $-0,25 \pm, 028$ & $-0,38 \pm, 111$ & $-0,26$ & $-0,27 \pm, 013$ & $-0,12 \pm, 082$ \\
EN3-7:EW & $-0,31$ & $-0,40 \pm, 046$ & $0,27 \pm, 134$ & $-0,33$ & $-0,27 \pm, 052$ & $0,10 \pm, 089$ \\
\hline
\end{tabular}

Die additiv-genetischen Korrelationen $r_{g}$ (add) und $r_{a}$ geschätzt mit beiden Modellen besitzen sehr hohe Ähnlichkeit. Dagegen zeigten die additiv- und dominanz-genetischen Korrelationen teilweise unterschiedliches Niveau. Für die Merkmalskombination EN3-7 zu EW ergaben sich in den Linien A und D mit -0,40 und -0,27 moderat negative $r_{a}$-Werte während die zugehörigen Werte von $r_{d}$ mit 0,27 und 0,10 positiv ausfielen allerdings behaftet mit hohen Standardfehlern im Vergleich zu den $r_{a}$-Schätzungen aus Tabelle 3. Ebenso scheinen die tierspezifischen Dominanzeffekte für die Merkmale EN1-2 und EN3-7 moderate positiv korreliert zu sein während die zugehörigen additiv-genetischen Effekte Korrelationen nahe Null aufweisen.

4. Zuchtwertschätzung

Durch den Übergang zu Dominanzmodellen im Kontext von Tiermodellen wird insbesondere eine genauere Schätzung für die Zuchtwerte erwartet. Bei der Vorhersage von additiven Effekten stellen die tierspezifischen Dominanzeffekte Störgrößen dar, die im rein-additiven Tiermodell zwangsläufig Bestandteil der Resteffekte sind. Folglich ergeben sich für bestimmte Tiergruppen über die Dominanzverwandtschaft (z.B. zwischen Vollgeschwistern) korrelierte Resteffekte. Betrachtet man z.B. einen Index aus Vollgeschwisterleistungen, konstruiert mit und ohne Dominanz, so ergibt sich für die erwartete absolute Differenz der geschätzten Zuchtwerte ohne $\left(\hat{\boldsymbol{a}}_{i}\right)$ und mit $\left(\hat{\boldsymbol{a}}_{i}^{D}\right)$ Dominanz für ein Tier $i$ die Abschätzung:

$$
\boldsymbol{E}\left(\left(\hat{\boldsymbol{a}}_{i}^{D}-\hat{\boldsymbol{a}}_{i} \mid\right) \leq \sqrt{\frac{2 \cdot \boldsymbol{f}^{2}}{\left(\boldsymbol{h}^{2}+2 \cdot \boldsymbol{f}^{2}\right)}} \cdot \boldsymbol{k} \cdot \sigma_{f} \quad \text { mit } \quad \boldsymbol{f}^{2}=\sigma_{f}^{2} / \sigma_{p}^{2}=\left(\mathbf{0 . 2 5} \cdot \sigma_{d}^{2}\right) / \sigma_{p}^{2}\right.
$$

Hierbei sind: $f^{2}$ der Anteil der Familienvarianz an der phänotypischen Gesamtvarianz und $k$ eine Konstante, mit $k=1$ falls für Tier $i$ mit Zuchtwert $a_{i}$ keine Eigenleistung vorhanden ist und mit $k=2$ falls zusätzlich die Eigenleistung vorliegt. Je größer die Anzahl der Vollgeschwister desto näher kommt der Erwartungswert in (3) seiner oberen Schranke. Für wesentlich komplexere Verwandtschaftsstrukturen ergibt sich generell $\left(2,0 \cdot \sigma_{f}\right)$ als maximale Differenz der Zuchtwerte, geschätzt mit und ohne Dominanz (VARONA u.a., 1998). In der zitierten Arbeit werden absolute Veränderungen unter $\left(0,1 \cdot \sigma_{f}\right)$ als geringfügig, über $\left(0,5 \cdot \sigma_{f}\right)$ als erwähnenswert und über $\left(1,0 \cdot \sigma_{f}\right)$ als wichtig eingeschätzt.

Zwischen $\sigma_{f}$ und der additiv-genetischen Standardabweichung besteht der Zusammenhang $\sigma_{f}=(\boldsymbol{f} / \boldsymbol{h}) \cdot \boldsymbol{\sigma}_{a}$ Unter der Annahme $\boldsymbol{h}^{2}=\boldsymbol{d}^{2}$ folgt $\boldsymbol{\sigma}_{f}=0,5 \cdot \sigma_{a}$, so dass wichtige Veränderungen über einer halben genetischen Standardabweichung liegen. Tabelle 5 zeigt die relative Anzahl der Tiere mit geringfügigen, erwähnenswerten und wichtigen Veränderungen der Zuchtwerte beim Übergang vom rein-additiven zum Dominanzmodell. Für Linie A liegt der Anteil der Zuchtwerte mit Änderungen zwischen $0,5 \cdot \sigma_{f}$ und $1,0 \cdot \sigma_{f}$ für die Legeleistung bei $10 \%$ und für das Eigewicht bei $2 \%$ 
während gleichzeitig der Anteil noch größerer Änderungen unter einem Prozent bleibt. In Linie D konnten lediglich für die Merkmale EN3-7 und EW bei mehr als 10\% der Tiere Veränderungen gefunden werden die über $0,5 \cdot \sigma_{f}$ lagen. Die Prozentzahlen in Tabelle 5 zeigen eine deutliche Abhängigkeit vom geschätzten Anteil der Dominanzvarianz an der Gesamtvarianz. Je größer der Anteil der Dominanzvarianz und je geringer die zugehörige Heritabilität desto größer fallen die erwarteten absoluten Veränderungen der Zuchtwerte aus (vgl. auch Beziehung (3)).

Tabelle 5

Anteil der Tiere (in \%), deren Zuchtwerte im additiven und Dominanzmodell vorgegebene Änderungen einhalten oder überschreiten (Percentage of animals with given changes of estimated breeding values when dominance was added to the models)

\begin{tabular}{l|ccc|c|c|c|c|c}
\hline & \multicolumn{3}{|c|}{ Linie A $(\mathbf{N}=\mathbf{1 0 0 9 9})$} & \multicolumn{4}{c}{ Linie D (N=10540) } \\
\hline Merkm. & $<\mathbf{0 , 1}$ & $\mathbf{0 , 1 - 0 , 5}$ & $\mathbf{0 , 5 - 1 , 0}$ & $>\mathbf{1 , 0}$ & $<\mathbf{0 , 1}$ & $\mathbf{0 , 1 - 0 , 5}$ & $\mathbf{0 , 5 - 1 , 0}$ & $>\mathbf{1 , 0}$ \\
\hline EN1-2 & 27,4 & 61,4 & 10,9 & 0,3 & 39,6 & 57,7 & 2,6 & 0,1 \\
EN3-7 & 29,1 & 59,7 & 10,5 & 0,7 & 29,5 & 58,9 & 10,5 & 1,1 \\
EN1-7 & 26,6 & 61,8 & 10,9 & 0,7 & 32,3 & 61,7 & 5,8 & 0,2 \\
EW & 39,5 & 58,4 & 2,1 & 0,0 & 24,5 & 62,6 & 12,5 & 0,4 \\
\hline
\end{tabular}

Die in Tabelle 6 dargestellten Rangkorrelationen für die 100 bzw. 50 besten Hennen auf jeder Generation belegen, dass die Veränderungen der Zuchtwerte geschätzt mit rein-additiven und mit Dominanzmodellen auch mit Rangverschiebungen verbunden sind. Werden die Zuchtwerte aller Hennen durch gewichtete Zusammenfassung über alle 3 Generationen herangezogen, so ergeben sich sehr hohe Werte nahe Eins. Verwendet man auf jeder Generation nur die 100 besten Hennen, gemäß ihrer Zuchtwerte aus dem Dominanzmodell, so liegen die gepoolten Korrelationen für EN1-2 und EW in Linie A bei 0.946 und 0.991 sowie in Linie D bei 0,996 und 0,951. In beiden Linien ergaben sich Rangkorrelationen unter 0,9 lediglich für das Merkmal EN3-7. Dieses Merkmal lieferte bei Betrachtung beider Linien auch die größten Veränderungen in den geschätzten Zuchtwerten beim Übergang zum Dominanzmodell (vgl. Tab. 5).

Tabelle 6

Rangkorrelationen zwischen den Zuchtwerten bei Verwendung von Tiermodellen mit und ohne Dominanz für die n-besten Hennen in jeder Generation (Rank correlations between breeding values using animal models with and without dominance for the best hens in each generation)

\begin{tabular}{l|c|c|c|c|c|c|c|c}
\hline \multirow{2}{*}{ Linie } & \multicolumn{4}{|c|}{$\mathbf{n = 1 0 0}$} & \multicolumn{4}{|c}{$\mathbf{n = 5 0}$} \\
\cline { 2 - 9 } & EN1-2 & EN3-7 & EN1-7 & EW & EN1-2 & EN3-7 & EN1-7 & EW \\
\hline A & 0,946 & 0,896 & 0,945 & 0,991 & 0,940 & 0,895 & 0,917 & 0,986 \\
D & 0,996 & 0,899 & 0,971 & 0,951 & 0,991 & 0,826 & 0,961 & 0,918 \\
\hline
\end{tabular}

Wie erwartet ziehen geringe Anteile von Dominanzvarianz für ein Merkmal nur geringe Änderungen der Zuchtwerte und folglich sehr hohe Korrelationen zwischen den entsprechenden Zuchtwerten nach sich.

\section{Diskussion}

Obwohl die untersuchten Linien intensiver züchterischer Bearbeitung unterliegen und sich durch hohes Leistungspotential auszeichnen, konnten Dominanzanteile an der Gesamtvarianz in Linie A für die Eizahl über 12\% und in Linie D für das Eigewicht über $17 \%$ ermittelt werden. Diese Tatsache ist jedoch verständlich, wenn man bedenkt, dass die untersuchten Linien in einem Kreuzungsprogramm stehen und Heterosis auftritt, falls Dominanzvariation und unterschiedliche Allelfrequenzen vorliegen. Die Verhältnisse und Größenordnungen der $\mathrm{d}^{2}$-Werte in Linie A für die Merkmale Eizahl 
und Eigewicht entsprechen den bisherigen Literaturergebnissen. So untersuchten WEI und VAN DER WERF (1993) drei Linien des Weißen Leghorns erstmalig mit einem Tiermodell und der REML-Methode. Die Autoren fanden für die Eizahl in Lebenswoche 18 bis 25 Dominanzanteile zwischen 10 und 19\% sowie für die Eizahl in Woche 18 bis 65 Werte zwischen 15 und 18\%. Die Dominanzanteile für die Eigewichte lagen in allen Linien unter den Anteilen der Eizahlen und variierten zwischen 1 und 13\%. BESBES und GIBSON (1999) analysierten simultan Reinzucht- und Kreuzungsleistungen von Legehennen aus 4 Generationen erfasst zwischen Lebenswoche 19 und 54. Die Schätzung der Varianzkomponenten erfolgte einerseits mit der Methode $\mathrm{R}$ angewendet auf ein Tiermodell und andererseits mit der „Tilde-hat“ Approximation bei Verwendung eines Vater-Mutter Modells. Die Dominanzanteile variierten in Abhängigkeit von der Schätzmethode für die Eizahl zwischen 4 und 16\% sowie für die Eigewichte zwischen 5 und 10\%. Untersuchungen mit großen Datensätzen von Milchrindern (MISZTAL u.a., 1998), von Fleischrindern (GENGLER u.a., 1997) und von Schweinen (CULBERTSON u.a., 1998) ergaben für einige Merkmale ebenfalls Dominanzanteile über 10\%. Die Betrachtung der Rangkorrelationen zwischen den Zuchtwerten geschätzt mit und ohne Dominanz lieferte für alle Tiere, für die 100 besten Tiere und für die 50 besten Tiere auf jeder Generation in Abhängigkeit der Dominanzanteile für EN1-7 Werte von 0,998, 0,945 und 0,917 in Linie A und Werte von 0,999, 0,971 und 0,961 in Linie D. Lediglich für das Merkmal EN3-7 mit h2- und d $\mathrm{d}^{2}$ Werten zwischen 12 und 16\%, also auf etwa ähnlichem Niveau, ergaben sich Rangkorrelationen unter 0,9 in beiden Linien.

Durch den Übergang zu Dominanzmodellen wird insbesondere für die zur Weiterzucht interessanten Tiere eine genauere Zuchtwertschätzung erreicht. Bei der Schätzung der Zuchtwerte stellen die tierspezifischen Dominanzeffekte Störgrößen dar, deren Ausschaltung besser gelingt, falls die Dominanzverwandtschaft bei den genetischen Berechnungen Berücksichtigung findet. Denkt man an die Erhöhung des Zuchtfortschrittes, so wird dieser nur gering ausfallen, falls der Anteil der Dominanzvarianz gering ist, die Heritabilitäten mittleres Niveau besitzen und ausschließlich auf die Verwendung der additiven Effekte orientiert wird. Allerdings fällt dieser Zuchtfortschritt in jeder Generation an und der Extragewinn ist gratis zu haben, ohne die Erfassung einer einzigen zusätzlichen Leistung. Untersuchungen von VARONA u.a. (1998) ergaben mit Daten von rund 3 Millionen Milchrindern Rangkorrelationen >0,999 zwischen den Zuchtwerten geschätzt mit und ohne Dominanz im Modell. Allerdings fanden die Autoren teilweise erhebliche Veränderungen in den geschätzten Zuchtwerten beim Übergang zu Dominanzmodellen, falls bestimmte Tiergruppen, z.B. charakterisiert durch die Anzahl der Vollgeschwister innerhalb der Nachkommenschaft, ausgewählt werden. Bei den Tierarten Schwein und Geflügel sollte die simultane Analyse von Reinzucht- und Kreuzungsleistungen unter Einbeziehung analytischer Modellrechnungen Gegenstand zukünftiger genetischer Berechnungen mit Dominanzmodellen sein.

\section{Literatur}

BESBES, B.; GIBSON, J.P.:

Genetic variation of egg production traits in purebred and crossbred laying hens. Animal Science 68 (1999), 433-439

BOX, G.E.P.; COX, D.R.:

An analysis of transformations. J. R. Statis. Soc. B, 26 (1964), 211-252 
CULBERTSON, M.S.; MISZTAL, I.; MABRY, J.W.; GENGLER, N.; BERTRAND, J.K.; VARONA, L.: Estimation and utilization of inbreeding and dominance genetic effects in genetic evaluation of purebred American swine. Proc. $6^{\text {th }}$ WCGALP (1998) Armidahl

DE BOER, I.J.M.:

Prediction of additive and dominance effects. EAAP, Edinburgh (1994)

GENGLER, N.; VAN VLECK, L.D.; MACNEIL, M.D.; MISZTAL, I.; PARIACOTE, F.A.:

Influence of dominance relationships on the estimation of dominance variance with sire-dam subclass effects. J. Anim. Sci. 75 (1997), 2885-2891

HOESCHELE, I.; VAN RADEN, P.M.:

Rapid inversion of dominance relationship matrices for noninbred populations by including sire by dam subclass effects. J. Dairy Sci. 74 (1991), 557-569

IBE, S.N.; HILL, W.G.:

Transformation of poultry egg production data to improve normality, homoscedasticity and linearity of genotypic regresssion. J. Anim. Breed. Genet. 105 (1988), 231-240

JARQUE, C.M.; BERA, A.K.

Efficiency tests for normality, heteroscedasticity and serial independence of regression residuals. Econ. Lett. 6 (1980), 255-259

KOERHUIS, A.N.M.:

Non-normality of egg production distributions in poultry and the effects of outlier elimination and transformation on size and curvilinearity of heritability. Livestock Production Science 45 (1996), 69-85

KOVAC, M.; GROENEVELD, E., GARCIA-CORTES, L.A.:

VCE-5, a package for the estimation of dispersion parameters. Proc. $7^{\text {th }}$ WCGALP (2002) Montpellier

LANGE, K.:

Mathematical and statistical methods for genetic analysis. Springer-Verlag New York, Inc. (1997)

MISZTAL, I.:

Estimation of variance components with large-scale dominance model. J. Dairy Sci. 80 (1997), 965-974

MISZTAL, I.; VARONA, L.; CULBERTSON, M.; GENGLER, N.; BERTRAND, J.K.; MABRY, J.;

LAWLOR, T.J.; VAN TASSELL, C.P.:

Studies on the value of incorporating effect of dominance in genetic evaluations of dairy cattle, beef cattle and swine. Proc. $6^{\text {th }}$ WCGALP (1998) Armidahl

UIMARI, P.; MÄKI-TANILA, A.:

Accuracy of genetic evaluations in dominance genetic models allowing for inbreeding. J. Anim. Breed. Genet. 109 (1992), 401-407

VARONA, L.; MISZTAL, I.; BERTRAND, J.K.; LAWLOR, T.J.:

Effect of full sibs on additive breeding values under the dominance model for stature in united states Holsteins. J. Dairy Sci. 81 (1998), 1126-1135

WEI, M.; VAN DER WERF, J.H.J.:

Animal model estimation of additive and dominance variances in egg production traits of poultry. J. Animal Science 71 (1993), 57-65

Eingegangen: 16.09.2002

Akzeptiert: 21.11.2002

Korrespondierender Autor

Dr. NORBERT MIELENZ

Institut für Tierzucht und Tierhaltung mit Tierklinik der

Martin-Luther-Universität Halle-Wittenberg

Adam-Kuckhoff-Straße 35

D-06108 Halle

E-Mail: mielenz@landw.uni-halle.de 